\title{
Use of root cause analysis in nursing education: Best practice from the quality and safety officer
}

\author{
Elizabeth Cooper *, Susan Pauly-O’Neill \\ University of San Francisco, San Francisco, United States
}

Received: February 19, 2015

Accepted: April 7, $2015 \quad$ Online Published: April 27, 2015

DOI: $10.5430 /$ jnep.v5n7p23

URL: http://dx.doi.org/10.5430/jnep.v5n7p23

\begin{abstract}
Teaching nursing students to be safe in practice is a key element to any nursing curriculum. This article will discuss the use of a Root Cause Analysis (RCO) framework with prelicensure nursing students, by the Quality and Safety Officer (QSO) in a School of Nursing and Health Professions, as a method to enhance transparency and improve patient safety. The aim is to provide a rationale for using this strategy, to identify the steps of a root cause analysis, to disclose barriers to its successful use, and to explore dissemination to the partnering healthcare environments.
\end{abstract}

Key Words: Root cause analysis, Quality and safety officer, Error reports, Transparency, Quality safety education for nurses

\section{INTRODUCTION}

Nurse educators have worked for more than a decade to address safety issues identified by the Institute of Medicine. ${ }^{[1]}$ Quality and safety edicts have been driving educational plans and influencing the experiences provided for nursing students in hopes of producing a safe, competent nurse. ${ }^{[2,3]}$ The American Association of Colleges of Nursing's Essentials of Baccalaureate Education for Professional Nursing Practice clearly states that the baccalaureate graduate must be prepared to promote safe, high quality patient care in a variety of healthcare settings. ${ }^{[4]}$ However, despite innumerable attempts to improve safety in the healthcare arena, errors continue to plague patients and providers. ${ }^{[5]}$ We have made little progress in improving the landscape; worldwide reports have stated that one in ten patients will experience an adverse incident including the leading risk of medical errors. ${ }^{[6]}$ For more than a decade, the adverse safety rate remains the same. ${ }^{[7]}$ This leaves the nurse educator with a difficult challenge. How can we create an environment that allows students the latitude to learn; yet provides sufficient checks and balances in a healthcare setting that remains unpredictable and potentially unsafe? Improving our prelicensure nursing students' rate of error reporting while encouraging transparency is one possible avenue. ${ }^{[8]}$

Transparency is a process that encourages members to share the truth without blame. ${ }^{[9]}$ The culture of transparency in healthcare gives the opportunity for providers, including nurses and nursing students, to increase their awareness of quality and safety issues in the healthcare setting. By increasing one's awareness through transparency, providers have full disclosure of the issues involving medical errors and can develop safer systems in response. ${ }^{[10]}$ Medical errors are events that affect the patient, which may fall into multiple categories. Two examples of possible categories are: plan of care not completed, tasks not executed correctly. ${ }^{[11]}$

\subsection{Background}

The quality and safety officer (QSO) is a formal role created to enhance safety and transparency in a school of nurs-

*Correspondence: Elizabeth Cooper; Email: eecooper@usfca.edu; Address: University of San Francisco, San Francisco, United States. 
ing and health professions in response to the call made by the Quality and Safety Education for Nurses (QSEN) initiative. ${ }^{[12]}$ QSEN's main mission supports the prelicensure student professional identity formation by encouraging faculty to include the knowledge, skills, and attitudes for six competencies: safety; informatics; teamwork and collaboration; quality improvement; evidence-based practice; and patient-centered care in the curriculum. ${ }^{[13]}$ The QSO role established at a private urban university has been successful in moving the School of Nursing and Health Professions toward a culture of safety reporting. Specifically, the QSO instituted a reporting system, using the root cause analysis framework. This initiative was developed to highlight the safety competency identified by QSEN and to facilitate a safety-reporting change in the School of Nursing and Health Professions.

Convening formal "Root Cause Analysis (RCA)" meetings to discuss medical errors sounds like a logical initial step. However, without first creating a culture in which this action feels constructive, such a gathering may feel punitive to the nursing student and instructor. Fear of retribution, failing a course, being removed from the nursing program and/or simple embarrassment are just a few of the reasons it is difficult for a nursing student to report an error. ${ }^{[14,15]}$ Adopting the use of a RCA framework within a culture of blame-free environment is one method nurse educators can use to create a constructive reporting environment. ${ }^{[16]}$

Health care environments have been notorious for dealing with errors by assigning blame. ${ }^{[15]}$ It is clear from the current error rate, the blame culture is not working. Instead, we can follow in the footsteps of other high-risk arenas like the automobile and aviation industries where the RCA was originally used to uncover systems problems. ${ }^{[7]}$ Utilizing the RCA systematic framework, which is defined as a systematic framework to facilitate open communication about an error, ${ }^{[14]}$ one can determine the cause of error. ${ }^{[17]}$ This is a process that encourages and allows all members involved in the error to discuss the event in a blame-free environment. The systematic approach searches for the what, when, why related to the error and then helps to develop recommendations that will prevent reoccurrences.

The process includes a formal error and near miss reporting system that dictates the use of a reporting tool to be used in addition to the reporting systems used in each partnering healthcare institution. ${ }^{[12]}$ The nursing student and nursing instructor report errors to the QSO. The QSO uses the RCA framework to provide a formal method of inquiry. The QSO reports back to the entire faculty and student body every semester to close the loop and perpetuate the cycle to decrease error rates over time.

\subsection{Safety reporting process}

We know that nurses, and nursing students under-report error. ${ }^{[15]}$ To enhance reporting, a culture of transparency and constructive analysis needed to be fostered. First steps included the introduction of a formal error reporting system developed and managed by the QSO. The policy is clearly outlined in each clinical syllabus, student handbook, faculty handbook, and the online learning management platforms accessible to both faculty and students. Students and faculty are instructed that this report must be completed in addition to any incident reports required by the health care unit.

When an error occurs in a clinical site, the student completes the internal error form within 48 hours. Incidents such as medication omission, wrong dose, patient fall, or needle stick are just a few examples. The QSO receives the error forms and over the course of the semester, analyzes the data looking for patterns. These patterns have included: not completing the entire patient rights protocol when administering medication; recapping needles; or discovering medicines in the wrong patient cassettes or bins. Uncovering these types of patterns is helpful as they are discussed at faculty meetings to put instructors on high alert and to ask classroom teachers to reiterate protocols. Along with examining patterns of errors, which help to identify gaps in our educational plan or system structures, the QSO conducts a root cause analysis in order to provide a safe environment in which to discuss each untoward event.

\section{ROOT CAUSE ANALYSIS}

The root cause analysis framework is used to identify the underlying reasons an error was made and to then move forward with recommendations to reduce the risk of recurrence. ${ }^{[18]}$ The goal is to provide an environment that is blame-free but safe enough to encourage taking responsibility for the error. It is important to use a systematic approach to ensure a fair and just process.

\subsection{RCA framework}

\subsubsection{Identification of RCA participants}

It is important to include the appropriate participants in the RCA to ensure that all sides are represented. Participants include: the nursing student, clinical instructor, RN working with student, unit manager, and the chair of the academic department. The crucial participants are the student and instructor and at a minimum these two people must be present to hold the RCA. The nurse working with the student and the unit manager also are invited. Extending this invitation solidifies how serious the university takes this event and also demonstrates our desire to work together.

ISSN 1925-4040 E-ISSN 1925-4059 
Table 1. Root cause analysis framework

\begin{tabular}{lll}
\hline Steps & Title & Description \\
\hline I & Inviting Participants & QSO receives and evaluates error form. Invites participants. \\
II & Environment & QSO sets up appropriate meeting environment. \\
III & Discussion & QSO facilitates blame-free discussion. \\
IV & Conclusion & QSO summarizes findings. \\
\hline
\end{tabular}

\subsubsection{Facilitating the $R C A$}

The RCA process can be nerve-wracking for the participants, especially for the student who made the error. Usually this is a first time event for all, making the participants anxious about the process. It is the QSO's job to make sure the process goes smoothly. This includes making sure the participants understand the process: ensuring that process rules are understood and followed.

\subsubsection{Process sequence}

Step I: Inviting Participants Once the error has been identified and reviewed by the QSO, the participants are invited to the RCA.

Step II: Setting up the Environment Setting up the environment for the meeting is important to success. The QSO is willing to conduct the RCA at the healthcare setting or at the university depending on the needs of the participants. Ideally, the QSO secures a conference room that is private, quiet, and offers an inclusive feel. A round or oval table with comfortable chairs provides an equal setting for all the participants.

Step III: Discussion The QSO begins the meeting with introductions and an explanation of the RCA process. All members are assured that the intent of the meeting is to create an open and fair dialogue to determine the etiology of an error. The QSO will manage the flow of the questions in order to ensure that each member is heard. The questions are focused on getting to the crux of the error and identifying all factors that played a role in the event. Patient identifiers are not included in the University driven reporting tool.

The student is the first to reflect. Questions asked by the QSO are:

(1) Will you please tell us what happened?

(2) What should have happened or what should you have done?

(3) What was the main cause?

(4) Do you feel you are safe in clinical?

During each response the QSO may ask follow-up questions to clarify. Next, the clinical faculty is asked to add any additional pertinent information. Then the same is asked of the RN, nurse manager, and Chair of the academic department.
Step IV: Conclusion Once the factors have been identified, it is the QSO's responsibility to summarize what has been presented. Restating what could have been done to avoid error is important. Coming to a conclusion with all participants as to the cause is important in order to have closure of this event. Not blaming the individual is key. Causes can be categorized to help guide a recommendation. Categories could include: system problem; environmental issue; knowledge deficient; communication factor; documentation factor; skill deficient; or human factors such as a lapse in memory.

Having the student re-state ways to avoid the error is important. It is also helpful to discuss factors that contributed to the issue. Concluding with a plan for the student that is simple and clear is the goal. The hope is that the outcome of the RCA includes the student returning to their original clinical site with a specific plan that encourages a feeling of confidence. The clinical plan may include: instructions on what type of patient to care for, when to call the instructor, and what specifically to study before arriving at the clinical site.

\subsection{Case studies}

Several case studies are presented to illustrate the RCA process. The case studies provide a descriptive scenario of the interactions of the RCA members. The sample case studies will help to familiarize readers with the RCA process. The cases are composites of several similar events and are presented only as instructional models.

\subsubsection{Medication error}

The QSO received an error report from a senior-level nursing student that the incorrect dose of a medication was administered.

Step I: The QSO read the report and invited participants to a RCA meeting. Potential attendees included the student, instructor, RN working with the student, nurse manager, and Chair of the Bachelor's in Nursing Department. The student, instructor, and Chair participated. The manager and RN declined the invitation.

Step II: The QSO obtained a quiet conference room with an oval table and comfortable chairs. 
Step III: The QSO facilitated the meeting. The session started with the QSO introducing the format of the RCA plus the intent of the meeting, which was to encourage open and fair dialogue. The student was first asked to "Please tell us what happened."

I was working with a nurse and had two patients to care for pretty much by myself. My nurse and I were to give our 9 am medications. I thought I was to give $200 \mathrm{mg}$ of thiamine IV. That is what I saw on the medication record so I drew up the $2 \mathrm{ml}$ of medication in a syringe. Then I drew up oral medications, crushed them, and put them in applesauce to give to the patient. I went into the patient's room and administered the thiamine. Next, I went to the bar code to scan the medication. At that time, I noticed I was to give 100 $\mathrm{mg}$ of thiamine and not $200 \mathrm{mg}$. I had misread the amount in the medication room.

What should you have done? I should have scanned the medication first.

Please tell me how the bar coding system works:

The computer has the medication record for each patient on it. You bring it to the pyxsis where the medications are stored. You remove the correct medications from the pyxsis using the computer's medical record. Then you bring the computer into the patient's room, complete the patient's rights, scan the medication, give the medication, and then chart the medication.

So what happened in this case?

I did not follow the procedure. I did complete the patient rights but I did not continue with the process. I gave the medication before I scanned it. If I had scanned it I would have seen that I was to give $100 \mathrm{mg}$ and not $200 \mathrm{mg}$. I skipped a step. When I first drew up the medication, I saw $200 \mathrm{mg}$. I have no answer as to why that happened.

The QSO now asked the clinical faculty if they have anything to add:

The student explained the incident correctly. The policy was not followed and that led to this error. The student did tell the RN right away and called me to let me know. The one other factor that I have witnessed is that I believed the student and RN preceptor were too casual with each other. I worried that the guidance was less because of this casual feeling. I talked to the student about this in the past. The student and $\mathrm{RN}$ should have looked at the computer together, to ensure the correct dose was prepared.

Step IV: Inexperience and not following the procedure led to this error. Why the student saw $200 \mathrm{mg}$ in the medication room was not clear. Lapse in memory does not seem to be the cause. The student read the record incorrectly but why is still unknown. The student appeared to have learned from this incident and both the instructor and student felt that the student was safe to return to the clinical setting. The student stated that they would discuss feeling too comfortable with the RN preceptor to ensure proper guidance. The student left the meeting with a sense of confidence and returned to clinical.

\subsubsection{Scope of practice error}

The QSO was called by a nursing instructor and informed that a junior student had disconnected a central line on a patient independently. The error form was sent to the QSO.

Step I: The QSO read the report and set the RCA framework into action. Participants were invited to the RCA. This included the student, instructor, RN working with student, manager, and chair of department. The student, instructor, chair of department, and manager of unit participated in RCA.

Step II: The QSO offered to facilitate the meeting in the healthcare setting if the manager wished. However, the manager preferred to come to the university setting. A private, quiet conference room with an oval table and comfortable chairs was obtained.

Step III: The QSO facilitated the meeting. The session started with the QSO introducing the format of the RCA plus the intent of the meeting, which was to encourage open and fair dialogue. The student was first asked to please tell us what happened.

I was working with my RN to care for one patient on an oncology unit. The patient had a double lumen central line. One port had normal saline at $30 \mathrm{cc} /$ hour infusing and the other line was clamped. I was alone in the room with my patient when the doctor came in and said the patient needed to go to $\mathrm{x}$-ray right away and to "discontinue the normal saline". Then, the transport person came in and said the patient had to go right away and told me to get the patient ready. He asked if I could disconnect the line. I 
went outside to the hall and could not find my nurse. The doctor came back and said "hurry up and get his patient down to x-ray". The doctor seemed angry, so I disconnected the central line and put a cap on the line.

How did you cap the line? I just disconnected the fluid line and put a blue cap on the end of the central line.

Did you put anything in the line like heparin or saline? No, I just capped the line and they took the patient to $x$-ray.

Then what happened?

They took the patient to x-ray. Then I went and found my nurse. I told her what happened and what I had done to the line. She was not happy. She explained that the line needed to be flushed with heparin. She told the manager what happened and went to x-ray to fix the line. I called my instructor.

What should have happened?

I should have stopped and found my nurse or instructor to help me. I knew it was wrong to disconnect the line but I felt so much pressure from the doctor. I wish I would have just said no but I wanted to make everyone happy.

The QSO asked the instructor if they had anything to add:

The account was correct. The one thing I would add is that there was a family member in the room who was also pushing for the student to disconnect the line. Everyone just wanted the $\mathrm{X}$-ray completed. When this was happening, I was on another floor with a different student so I did not know this student needed help. The student did call me and knew right away that this was outside of the scope of practice. The student has always been well prepared and acted appropriately. This is a first time event.

The QSO asked the manager to respond:

I was very unhappy to hear that a nursing student acted out of their scope of practice. My patients are very sick and disconnecting a line can be devastating. The student and instructor did show appropriate concern and sorrow for this event. I need to know that it will not happen again. With that said, I did not know that you had people pressuring you to disconnect this line. I am not happy to hear that the doctor was pushing you that way. It is good to discuss this so that I can bring this back so that you will not have this happen again. Also, I will talk to my staff and the transport staff about students' scope of practice.

I am so relieved to know that the university takes these events seriously and has a protocol to follow. I feel so much better being a part of this discussion.

QSO asked the student: do you feel safe in clinical:

Yes, I do feel safe. If anything I am safer because I clearly know my scope of practice. I will always call my nurse or instructor. This has been so scary and I feel terrible this happened but I will now be safe.

The instructor and manager agreed that the student would be safe back in clinical. Additionally, both agreed they would help any time if needed.

Step IV: Inexperience, inability to say no, wanting to please people, and not understanding scope of practice all led to this error. Wanting to please people of a perceived higher rank was a human factor. Judgment error plays a role in this event. The student, instructor and manager all felt that this student could return to the clinical setting.

These two examples of RCA in action demonstrated the power of talking about the event in detail. All members of the analysis walked away with a better knowledge of the factors that led to the error. Having the student felt heard and believed was imperative. Providing a forum that allowed the participants to speak freely, honestly, and blame-free was truly a gift that educators offered.

\section{Disseminating THE RCA FRAMEWORK}

Utilizing the RCA framework in the academic setting proved successful with the prelicensure nursing students. The above case studies demonstrated the process. Can the process disseminate to other settings? The RCA framework provided by the academic setting, the process for root cause analysis was successfully piloted in an outpatient setting. To highlight its use, a case scenario is offered.

\section{Outpatient pediatric respiratory distress case study}

A child in respiratory distress left a clinic waiting area to travel by car to an emergency department. Although the clinic had a policy dictating that these patients bypass registration and be taken directly into an examination room for 
initial treatment, it was not followed due to miscommunication. Clinic administrators suggested immediate punitive action, but were encouraged to consider convening their first root cause analysis. The supervising nurse practitioner served in the QSO role at the outpatient clinic.

In order to facilitate open, constructive communication aimed at quality improvement, a RCA was planned and facilitated by the supervising nurse practitioner. Allowing staff to discuss the event in a blame-free environment was proposed as a better strategy to find solutions to a breakdown in the transport policy. Participating staff were reassured that the discussion would revolve around potential contributing factors such as knowledge related to the patient's condition or lack of understanding of the policy. A diagram of human error management using coaching was offered as a starting point. Everyone was comfortable that after determining the root cause, recommendations for improvement would be discussed. The registration tech, medical assistant, nurse practitioner, physician, and medical director all agreed.

Interesting dialogue resulted which provided new insight from many angles. From the vantage point of the front desk, questions arose about whether patients could be taken from the waiting area without first providing basic demographic information. From the medical assistant lens, concern about summoning a nurse practitioner or physician into the waiting area surfaced. Both the nurse practitioner and physician were unclear about how best to insist that the family bring the child into an examination room when they preferred to go to an emergency department. So what seemed like a straightforward policy, was not so simple after all. The so- lution was assessment of the situation from all viewpoints, education regarding potential rapid patient deterioration, and role-playing likely scenarios. Punitive action was avoided and safety for future patients was improved.

\section{Conclusion}

Creating a culture of safety that allows nursing students to learn is a difficult task for the nurse educator. Enhancing safety reporting may increase transparency and led to improved patient safety. Developing the role of a Quality and Safety Officer whose goal is to make changes involving the safety culture at the School of Nursing and Health Professions is one method to help the nurse educator and the nursing students. Utilizing the root cause analysis method is one way that nurse educators can use to help facilitate open communication about an error. This step-by-step process leads to open communication, which fosters the creation of solutions to medical errors instead of simply placing blame. The case studies demonstrated the value of the RCA in an academic setting with prelicensure nursing students and nursing instructors. Dissemination of the RCA process to the outpatient setting highlighted its value in multiple settings with an advanced practice nurse. Putting patient safety first must be the goal of all healthcare providers. Helping our nursing students learn early in their educational process about safety reporting, blame free environments, and responsibility may increase safety at the bedside.

\section{CONFlicts OF INTEREST Disclosure}

The authors declare that there is no conflict of interest statement.

\section{REFERENCES}

[1] Institute of Medicine. Health professions education: A bridge to quality. Washington, DC: National Academic Press; 2003.

[2] Benner P, Stuphen M, Leonard V, et al. Educating nurses: A call for radical transformation. San Francisco, CA: Jossey-Bass; 2009.

[3] Cooper E, Prion S, Pauly-O'Neill S. Comparison of student experience with critical events during simulation and acute care hospital rotations. Nurse Educator. 2015; 40(1): 31-35. PMid:25127080 http://dx.doi.org/10.1097/NNE.0000000000000075

[4] American Association of Colleges of Nursing. The essentials of baccalaureate education for professional nursing practice. Washington, DC. 2008.

[5] Wachter R. Patient safety at ten: Unmistakable progress, troubling gaps. Health Affairs. 2010; 29(1): 165-173. PMid:19952010 http://dx.doi.org/10.1377/hlthaff.2009.0785

[6] Tella S, Liukka M, Jamookeeah D, et al. What do nursing students learn about patient safety? An integrative literature review. Journal of Nursing Education. 2014; 53(1): 7-13. PMid:24308538
[7] Hettinger A, Fairbanks R, Hegde S, et al. An evidence-based toolkit for the development of effective and sustainable root cause analysis system safety solutions. American Society for Healthcare Risk Management. 2013; 33(2): 11-20.

[8] Hession-Laband E, Mantell P. Lessons learned: Use of event reporting by nurses to improve patient safety and quality. Journal of Pediatric Nursing. 2011; 26: 149-155. PMid:21419975 http: //dx.doi.org/10.1016/j.pedn.2010.12.005

[9] Paterick Z, Paterick B, Waterhouse B, et al. The challenges to transparency in reporting medical errors. Journal of Patient Safety. 2009; 5: 205-209. PMid:22130212 http://dx.doi.org/10.1097/PTS $.0 \mathrm{~b} 013 \mathrm{e} 3181 \mathrm{be} 2 \mathrm{a} 88$

[10] Sherwood G, Drenkard K. Quality and safety curricula in nursing education: Matching practice realities. Nursing Outlook. 2007; 55: 151-155. PMid:17524803 http://dx.doi.org/10.1016/j.out look. 2007.02.004

[11] Institute of Medicine. To err is human: Building a safer health system. Washington, DC: National Academic Press. 1999. 
[12] Cooper E. Establishing the role of a quality and safety officer in a school of nursing. In L. Caputi (Ed). Building the Future of Nursing. Baltimore, MD: Wolters Kluwer Lippincott Williams \& Wilkins. 2014: 45-50

[13] Cronenwett L, Sherwood G, Barnsteiner J, et al. Quality and safety education for nurses. Nursing Outlook. 2007; 55(3): 122-131. PMid:17524799 http://dx.doi.org/10.1016/j.outlook.2007.02.006

[14] Cooper E. From the school of nursing quality and safety officer: Nursing students' use of safety reporting tools and their perception of safety issues in clinical settings. Journal of Professional Nursing. 2013; 29(2): 109-116. PMid:23566457 http://dx .doi.org/10. 1016/j.profnurs. 2012.12.005
[15] Sherwood G, Barnsteiner J. Safety. Barnsteiner (Eds.) Quality and Safety in Nursing: A Competency Approach to Improving Outcomes. West Sussex, UK: Wiley-Blackwell. 2012: 149-169.

[16] Vottero B. Immersion into a root cause analysis. In L. Caputi (Ed) Building the Future of Nursing. Baltimore, MD: Wolters Kluwer Lippincott Williams \& Wilkins. 2014; 9-17.

[17] Percarpio K, Watts B, Weeks W. The effectiveness of root cause analysis: What does the literature tell us? The Joint Commission Journal on Quality and Patient Safety. 2008; 34(7): 391-398. PMid:18677870

[18] Ewen M, Bucher G. Root cause analysis: Responding to a sentinel event. Home Healthcare Nurses. 2013; 31(8): 435-443. http: //dx.doi.org/10.1097/NHH.0b013e3182a1dc32 